\title{
Assouad Dimensions and Lower Dimensions of Some Moran Sets
}

\author{
JiaQing Xiao $\mathbb{D}$ \\ Huanggang Normal University, HuangGang 438000, China \\ Correspondence should be addressed to JiaQing Xiao; 2280122005@qq.com \\ Received 17 July 2020; Revised 22 August 2020; Accepted 12 September 2020; Published 23 September 2020 \\ Academic Editor: Kit C. Chan \\ Copyright ( 2020 JiaQing Xiao. This is an open access article distributed under the Creative Commons Attribution License, which \\ permits unrestricted use, distribution, and reproduction in any medium, provided the original work is properly cited.
}

We prove that the low dimensions of a class of Moran sets coincide with their Hausdorff dimensions and obtain a formula for the lower dimensions. Subsequently, we consider some homogeneous Cantor sets which belong to Moran sets and give the counterexamples in which their Assouad dimension is not equal to their upper box dimensions and packing dimensions under the case of not satisfying the condition of the smallest compression ratio $c_{*}>0$.

\section{Introduction}

Let us begin with the definition of the Assouad dimension and the lower dimension. For $r>0, E \subseteq R^{d}$, and $N_{r}(E)$ denotes the smallest number of open sets required for an $r$ cover of a bounded set $E$.

Definition 1. The Assouad dimension of a nonempty set $F \subseteq R^{d}$ is defined by $\operatorname{dim}_{A} F=\inf \{\alpha \geq 0$. There exists a constant $c>0$ such that, for any $0<r<R$, and $x \in F$, $\left.N_{r}(B(x, R) \cap F) \leq c(R / r)^{\alpha}\right\}$.

If the Hausdorff dimension provides fine, but global, geometric information, then the Assouad dimension which was introduced by Assouad [1] provides coarse, but local, geometric information. The Assouad dimension is a fundamental notion of dimension used to study fractal objects in a wide variety of contexts. An important theme in dimension theory is that dimensions often come in pairs. The natural partner of the Assouad dimension is the lower dimension, which was introduced by Larman [2], where it was called the minimal dimensional number.

Definition 2. The lower dimension of $\mathrm{F}$ is defined by $\operatorname{dim}_{L} F=\inf \{\alpha \geq 0$. There exists a constant $c>0$ such that, for any $0<r<R \leq|F|$, and $\left.x \in F, N_{r}(B(x, R) \cap F) \geq c(R / r)^{\alpha}\right\}$.

The lower dimension is not monotonic, and the modified lower dimension is defined by

$$
\operatorname{dim}_{\mathrm{ML}} F=\sup \left\{\operatorname{dim}_{L} E: E \subseteq F\right\} .
$$

The Assouad dimension has recently received an enormous interest in the mathematical literature due to its connections with the doubling property. This lead Larman to introduce the dual notion of dimension, namely, the lower Assouad dimension, often simply called the lower dimension. Just like the Assouad dimension, the lower dimension has also received an enormous interest in the mathematical literature due to its connections with the uniform property of metric spaces. As a result of this, a large number of papers have investigated the Assouad dimension and the lower dimension of different classes of fractal sets. Olsen [3] gave a simple and direct proof that the Assouad dimension of a graph-directed Moran fractal satisfying the open-set condition which is Ahlfors regular coinciding with its Hausdorff and box dimensions. However, in general, it is difficult to obtain the Assouad dimensions of sets which are not Ahlfors regular. Mackay [4] calculated the Assouad dimension of the self-affine carpets of Bedford and McMullen and his main result solved the problem posed by Olsen [3]. For the Moran sets introduced by Wen [5] which are not Ahlfors regular, Li et al. [6] obtained the Assouad dimensions of Moran sets under suitable condition and studied the Assouad dimensions of Cantor-like sets. Jinjun Li [7] also show that the Assouad dimensions of some Moran sets coincide with their packing and upper box dimensions. However, Li [7] did not compute the lower dimension of this class of fractals, the main conclusions of the paper $[6,7]$ must satisfy the 
condition that the smallest compression ratio $c_{*}>0$ and the paper [7] conjecture that the conclusion remains true if the condition $c_{*}>0$ is removed (see Remark 1 [7]). In this paper, we prove that the low dimensions of a class of Moran sets coincide with their Hausdorff dimensions and obtain a formula for the lower dimensions. Subsequently, we consider some homogeneous Cantor sets which belong to Moran sets and give the counterexamples which their Assouad dimension is not equal to their upper box dimensions and packing dimensions under the case of not satisfying the condition that the smallest compression ratio $c_{*}>0$, and we give a negative answer to the conjecture in the paper [7].

\section{Lower Dimensions of Some Moran Sets}

Firstly, let us recall the definition of Moran sets introduced by Wen [5]. Let $\left\{n_{k}\right\}_{k \geq 1}$ be a sequence of positive integers. Define $D_{0}=\varnothing$, and for any $k \geq 1$, set $D_{m, k}=\left\{\left(i_{m}, i_{m+1}, \ldots, i_{k}\right) ; 1 \leq i_{j} \leq n_{j}, m \leq j \leq k\right\}, \quad D_{k}=D_{1, k}$, and $D=\cup_{k \geq 0} D_{k}$. If $\sigma=\left(\sigma_{1}, \sigma_{2}, \ldots, \sigma_{k}\right) \in D_{k}, \tau=\left(\tau_{1}, \ldots, \tau_{m-k}\right) \in D_{k+1, m}$, let $\sigma * \tau=\left(\sigma_{1}, \ldots, \sigma_{k}, \tau_{1}, \ldots, \tau_{m-k}\right)$. And if $\sigma \in D_{k}$, remark $\sigma \mid l=\left(\sigma_{1}, \ldots, \sigma_{l}\right)$ for $1 \leq l \leq k$.

Definition 3. Suppose that $J \subset R^{d}$ is a compact set with $\overline{\text { int } J}=J$. Let $\left\{\phi_{k}\right\}$ be a sequence of positive real vectors with $\left\{\phi_{k}\right\}=\left(c_{k, 1}, c_{k, 2}, \ldots, c_{k, n_{k}}\right)$ and $\sum_{j=1}^{n_{k}} c_{k, j} \leq 1, k \in N$. We say the collection $F=\left\{J_{\sigma} ; \sigma \in D\right\}$ of closed subsets of $J$ fulfills the Moran structure if it satisfies the following Moran structure conditions (MSC):

(1) For $\sigma \in D, J_{\sigma}$ is geometrically similar to $J$, i.e., there exists a similarity $S_{\sigma}: R^{d} \longrightarrow R^{d}$ such that $J_{\sigma}=S_{\sigma}(J)$. For convenience, we write $J_{\varnothing}=J$

(2) For all $k \geq 0$ and $\sigma \in D_{k}, J_{\sigma * 1}, J_{\sigma * 2}, \ldots, J_{\sigma * n_{k+1}}$ are subsets of $J_{\sigma}$ and satisfy that int $J_{\sigma * i} \cap$ int $j_{\sigma * j}=\varnothing(i \neq j)$

(3) For any $k \geq 1$ and $\sigma \in D_{k-1}, 1 \leq j \leq n_{k}$,

$$
\frac{\left|J_{\sigma * j}\right|}{\left|J_{\sigma}\right|}=c_{k, j},
$$

where $|A|$ denotes the diameter of $A$.

Suppose that $F$ is a collection of closed subsets of $J$ fulfilling the Moran structure, set

$$
\begin{aligned}
E_{k} & =\bigcup_{\sigma \in D_{k}} J_{\sigma}, \\
E & =\bigcap_{k \geq 0} E_{k} .
\end{aligned}
$$

It is ready to see that $E$ is a nonempty compact set. The set $E=E(F)$ is called the Moran set associated with the collection $F$.

Let $F_{k}=\left\{J_{\sigma} ; \sigma \in D_{k}\right\}$ and $F=\cup_{k \geq 0} F_{k}$. The elements of $F_{k}$ are called kth-level basic sets of $E$ and the elements of $F$ are called the basic sets of $E$. Suppose that the set $J$ and the sequences $\left\{n_{k}\right\}$ and $\left\{\phi_{k}\right\}$ are given. We denote by
$M=M\left(J,\left\{n_{k}\right\},\left\{\phi_{k}\right\}\right)$ the class of the Moran sets satisfying the MSC. We call $M\left(J,\left\{n_{k}\right\},\left\{\phi_{k}\right\}\right)$ the Moran class associated with the triplet $\left(J,\left\{n_{k}\right\},\left\{\phi_{k}\right\}\right)$.

Remark 1. From the above definition, we see that if the Moran sets $E_{1}, E_{2} \in M\left(J,\left\{n_{k}\right\},\left\{\phi_{k}\right\}\right)$ and $E_{1} \neq E_{2}$, then the relative positions of $k^{\text {th }}$-level basic sets of $E_{1}$ and those of $E_{2}$ may be different, although they satisfy the same MSC.

Under some mild conditions, Hua et al. [8] gave the Hausdorff packing and upper box dimensions of Moran sets. To state their result, we need some notations. Let $M=M\left(J,\left\{n_{k}\right\},\left\{\phi_{k}\right\}\right)$ be a Moran class. Let $c_{*}$ : inf $c_{i, j}$ and $c_{\sigma}=c_{1, \sigma_{1}}, \ldots, c_{k, \sigma_{k}}$ for $\sigma=\sigma_{1}, \ldots, \sigma_{k} \in D_{k}$. Let

$$
\begin{aligned}
& s_{*}=\liminf _{k \longrightarrow \infty} s_{k}, \\
& s^{*}=\limsup _{k \longrightarrow \infty} s_{k},
\end{aligned}
$$

where $s_{k}$ satisfies the following equation:

$$
\prod_{i=1}^{k} \sum_{j=1}^{n_{i}} c_{i, j}^{s_{k}}=\sum_{\sigma \in D_{k}} c_{\sigma}^{s_{k}}=1 .
$$

Set

$$
\begin{aligned}
& \underline{h}=\liminf _{k \longrightarrow \infty} \sum_{\sigma \in D_{k}}\left(J_{\sigma}\right)^{s_{*}}, \\
& \bar{h}=\limsup _{k \longrightarrow \infty} \sum_{\sigma \in D_{k}}\left(J_{\sigma}\right)^{s_{*}} .
\end{aligned}
$$

We can now present the main result of Hua et al. [8].

Theorem 1 (see [8]). Suppose that $M=M\left(J,\left\{n_{k}\right\},\left\{\phi_{k}\right\}\right)$ is a Moran class satisfying $c_{*}>0$. Then, for any $E \in M$,

$$
\begin{aligned}
\operatorname{dim}_{H} E & =s_{*}, \\
\operatorname{dim}_{P} E & =\overline{\operatorname{dim}}_{B} E=s^{*} .
\end{aligned}
$$

Li [7] computed the Assouad dimension of a fairly general (and important) class of Moran fractals.

Theorem 2 (see [7]). Suppose that $M=M\left(J,\left\{n_{k}\right\},\left\{\phi_{k}\right\}\right)$ is a Moran class satisfying $c_{*}>0$. Then, for any $E \in M$,

$$
\operatorname{dim}_{P} E=\overline{\operatorname{dim}}_{B} E=\operatorname{dim}_{A} E=s^{*} .
$$

The natural partner of the Assouad dimension is the lower dimension, and we prove that the low dimensions of a class of Moran sets coincide with their Hausdorff dimensions and obtain a formula for the lower dimensions.

Theorem 3. Suppose that $M=M\left(J,\left\{n_{k}\right\},\left\{\phi_{k}\right\}\right)$ is a Moran class satisfying $c_{*}>0$ and $0<\underline{h} \leq \bar{h}<\infty$.Then, for any $E \in M$,

$$
\operatorname{dim}_{L} E=\operatorname{dim}_{\mathrm{ML}} E=\operatorname{dim}_{H} E=s_{*} .
$$


Lemma 1. There exists a probability measure $v$ supported by the Moran E such that

$$
v\left(J_{\sigma_{0}}\right)=\frac{\left(J_{\sigma_{0}}\right)^{s_{*}}}{\sum_{\sigma \in D_{k}}\left(J_{\sigma}\right)^{s_{*}}},
$$

for any $k \geq 1$ and $\sigma_{0} \in D_{k}$.

Proof. Take a sequence of probability measures $\left\{v_{m}\right\}_{m \geq 1}$ supported by $E$ such that

$$
v_{m}\left(J_{\sigma_{0}}\right)=\frac{\left(J_{\sigma_{0}}\right)^{s_{*}}}{\sum_{\sigma \in D_{m}}\left(J_{\sigma}\right)^{s_{*}}},
$$

for any $\sigma_{0} \in D_{m}$.

More precisely, we can construct $v_{m}$ as follows.

First, we distribute the unit mass among the $m^{\text {th }}$-level basic elements according to (11). Inductively, suppose that we have already distributed the mass of proportion $v_{m}\left(J_{\sigma}\right)$ to a $k^{\text {th }}$-level basic set $J_{\sigma}\left(\sigma \in D_{k}, k \geq m\right)$; then, we distribute the mass concentrated on $J_{\sigma}$ evenly to each of its $(k+1)$ th-level basic subsets, i.e.,

$$
v_{m}\left(J_{\sigma * j}\right)=\frac{\left(c_{k+1, j}\right)^{s_{*}}}{\sum_{l=1}^{n_{k+1}}\left(c_{k+1, l}\right)^{s_{*}}} v_{m}\left(J_{\sigma}\right),
$$

for $1 \leq j \leq n_{k+1}$.

Repeating the above procedure, we get the desired measure.

Now, fix some $m \geq 1$; for any $k<m$ and $\sigma_{0} \in D_{k}$, we obtain

$$
v_{m}\left(J_{\sigma_{0}}\right)=\sum_{\tau \in D_{k+1, m}} v_{m}\left(J_{\sigma_{0} * \tau}\right) .
$$

Combining it with (11), we have

$$
\left(\sum_{\sigma \in D_{m}}\left|J_{\sigma}\right|^{s_{*}}\right) v_{m}\left(J_{\sigma_{0}}\right)=\sum_{\tau \in D_{k+1, m}}\left|J_{\sigma_{0} * \tau}\right|^{s_{*}} .
$$

For any $\sigma_{1} \in D_{k}$, by the definitions of $E$,

$$
\frac{\left|J_{\sigma_{1} * \tau}\right|}{\left|J_{\sigma_{1}}\right|}=\frac{\left|J_{\sigma_{0} * \tau}\right|}{\left|J_{\sigma_{0}}\right|}
$$

and thus by (14),

$$
\left|J_{\sigma_{1}}\right|^{s_{*}}\left(\sum_{\sigma \in D_{m}}\left(J_{\sigma}\right)^{s_{*}}\right) v_{m}\left(J_{\sigma_{0}}\right)=\left|J_{\sigma_{0}}\right|^{s_{*}} \sum_{\tau \in D_{k+1, m}}\left|J_{\sigma_{1} * \tau}\right|^{s_{*}} .
$$

This gives

$$
\left(\sum_{\sigma_{1} \in D_{k}}\left|J_{\sigma_{1}}\right|^{s_{*}}\right)\left(\sum_{\sigma \in D_{m}}\left(J_{\sigma}\right)^{s_{*}}\right) v_{m}\left(J_{\sigma_{0}}\right)=\left|J_{\sigma_{0}}\right|^{s_{*}} \sum_{\sigma_{1} \in D_{k}, \tau \in D_{k+1, m}}\left|J_{\sigma_{1} * \tau}\right|^{s_{*}} \text {. }
$$

Observing that

$$
\sum_{\sigma \in D_{m}}\left(J_{\sigma}\right)^{s_{*}}=\sum_{\sigma_{1} \in D_{k}, \tau \in D_{k+1, m}}\left|J_{\sigma_{1} * \tau}\right|^{s_{*}}
$$

one obtains

$$
v_{m}\left(J_{\sigma_{0}}\right)=\frac{\left(J_{\sigma_{0}}\right)^{s_{*}}}{\sum_{\sigma \in D_{k}}\left(J_{\sigma}\right)^{s_{*}}} .
$$

To summarize, we obtain a sequence of probability measures $\left\{v_{m}\right\}_{m \geq 1}$ supported by $E$ and satisfy (10) for any $k \leq m$ and $\sigma_{0} \in D_{k}$.

Now, Hellys theorem [9] enables us to extract a subsequence $\left\{v_{m_{n}}\right\}_{n \geq 1}$ converging weakly to a limit measure $v$.

To verify that $v$ fulfills the desired requirements, we fix some $k \geq 0$ and $\sigma_{0} \in D_{k}$. Then, by the properties of the weak convergence,

$$
\varlimsup_{n \longrightarrow \infty} v_{m_{n}}\left(J_{\sigma_{0}}\right) \leq v\left(J_{\sigma_{0}}\right) .
$$

Combining with (19), this implies

$$
v\left(J_{\sigma_{0}}\right) \geq \frac{\left(J_{\sigma_{0}}\right)^{s_{*}}}{\sum_{\sigma \in D_{k}}\left(J_{\sigma}\right)^{s_{*}}} .
$$

On the other hand, take an $\varepsilon>0$ small enough so that the $\varepsilon$-neighborhood $J(\varepsilon)$ of $J_{\sigma_{0}}$ is separated from the other $m^{\text {th }}$-level basic set; then, $v_{m_{n}}(J(\varepsilon))=v_{m_{n}}\left(J_{\sigma_{0}}\right)$. By the properties of weak convergence, the following holds:

$$
\varliminf_{n \longrightarrow \infty} v_{m_{n}}(J(\varepsilon)) \geq v(J(\varepsilon)) \geq v\left(J_{\sigma_{0}}\right) .
$$

Combining with (19) yields

$$
v\left(J_{\sigma_{0}}\right) \leq \frac{\left(J_{\sigma_{0}}\right)^{s_{*}}}{\sum_{\sigma \in D_{k}}\left(J_{\sigma}\right)^{s_{*}} .}
$$

We have for any $k \geq 1$ and $\sigma_{0} \in D_{k}$,

$$
v\left(J_{\sigma_{0}}\right)=\frac{\left(J_{\sigma_{0}}\right)^{s_{*}}}{\sum_{\sigma \in D_{k}}\left(J_{\sigma}\right)^{s_{*}}} .
$$

Finally, for any $x$ which is not in $E$, since $E$ is a closed set, there exists an open set $\mathrm{U}$ containing $x$ and separated from $E$, and thus, $v(U) \leq \underline{\lim }_{n \rightarrow \infty} v_{m_{n}}(U)=0$, which asserts that $v$ is supported by $E$.

Lemma 2 (see $[1,2])$. If $F \subset R^{d}$ is closed, then

$\operatorname{dim}_{L} E \leq \operatorname{dim}_{\mathrm{ML}} E \leq \operatorname{dim}_{H} E \leq \operatorname{dim}_{P} E \leq \overline{\operatorname{dim}}_{B} E \leq \operatorname{dim}_{L} E$.

For $\sigma \in D$, we denote by $\sigma$ - the word obtained by deleting the last letter of $\sigma$. For $\gamma>0$, we define $\Gamma(\gamma)$ by $\Gamma(\gamma)=\left\{\sigma \in D \mid c_{\sigma}<\gamma \leq c_{\sigma-}\right\}$. 
Lemma 3 (see [7], Lemma 3.1). If $c_{*}>0$, there exists a constant $l_{0}$ such that $\#\left\{\tau \in \Gamma(\gamma) \mid B(x, \gamma) \cap J_{\tau} \neq \varnothing\right\} \leq l_{0}$ for all $x \in E$ and $\gamma>0$.

Remark 2. Some subtly different definitions of the low dimension are given as follows: $\operatorname{dim}_{L} F=\inf \{\alpha \geq 0$. There exist two constant $c>0$ and $b>0$ such that, for any $0<r<R<b \leq|F|$, and $\left.x \in F, N_{r}(B(x, R) \cap F) \geq c(R / r)^{\alpha}\right\}$.

It is easy to check this definition and Definition 2 coincides.

Proof of Theorem 3. Fix $x \in E$ and $0<r<R$; there exists $\sigma_{0} \in \Gamma(R /|J|)$ such that $x \in J_{\sigma_{0}}$ and $J_{\sigma_{0}} \subset B(x, R)$. By Lemma 1 , there exists a probability measure $v$ supported by $E$, such that

$$
\begin{aligned}
& v\left(J_{\sigma_{0}}\right)=\frac{\left(J_{\sigma_{0}}\right)^{s_{*}}}{\sum_{\sigma \in D_{\left|\sigma_{0}\right|} \mid}\left(J_{\sigma}\right)^{s_{*}}}, \\
& v\left(J_{\sigma_{0}}\right)=\sum_{\tau \in \Gamma(r|| J \mid), J_{\tau} \subset J_{\sigma_{0}}} v\left(J_{\tau}\right)=\sum_{\tau \in \Gamma(r|| J \mid), J_{\tau} \subset \subset J_{\sigma_{0}}} \frac{\left(J_{\tau}\right)^{s_{*}}}{\sum_{\sigma \in D_{|\tau|}}\left(J_{\sigma}\right)^{s_{*}}},
\end{aligned}
$$

where $\left|\sigma_{0}\right|$ denotes the rank of $J_{\sigma_{0}}$, i.e., $J_{\sigma_{0}}$ is the $\left|\sigma_{0}\right|^{\text {th }}$-level basic set. This implies that

$$
\frac{\left|J_{\sigma_{0}}\right|^{s_{*}}}{\sum_{\sigma \in D_{\left|\sigma_{0}\right|}\left|J_{\sigma}\right|^{s_{*}}}} \leq \#\left\{\tau \in \Gamma\left(\frac{r}{|J|}\right), J_{\tau} \subset J_{\sigma_{0}}\right\} \frac{r^{s_{*}}}{\sum_{\sigma \in D_{|\tau|} \mid}\left|J_{\sigma}\right|^{s_{*}}} .
$$

By Remark 2, let $R$ be small enough such that

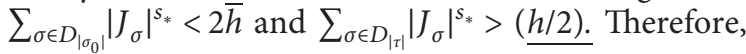

$$
\begin{aligned}
\#\left\{\tau \in \Gamma\left(\frac{r}{|J|}\right), J_{\tau} \subset J_{\sigma_{0}}\right\} & \geq\left(c_{*}\right)^{s_{*}} \frac{\sum_{\sigma \in D_{|\tau|} \mid}\left|J_{\sigma}\right|^{s_{*}}}{\sum_{\sigma \in D_{\left|\sigma_{0}\right|} \mid}\left|J_{\sigma}\right|^{s_{*}}}\left(\frac{R}{r}\right)^{s_{*}} \\
& >\left(c_{*}\right)^{s_{*}} \frac{\underline{h}}{4 \bar{h}}\left(\frac{R}{r}\right)^{s_{*}} .
\end{aligned}
$$

Using Lemma 3 and (28), we attain

$$
N_{r}(B(x, R) \cap E)>\frac{\left(c_{*}\right)^{s_{*}}}{l_{0}} \frac{\underline{h}}{4 \bar{h}}\left(\frac{R}{r}\right)^{s_{*}},
$$

which implies $\operatorname{dim}_{L}(E)>s_{*}$ and by Lemma 2 and Theorem 1 , the proof of Theorem 3 is completed.

\section{Assouad Dimensions and Lower Dimensions of Homogeneous Cantor Set}

Definition 4. Suppose that $J$ is the interval $[0,1]$ and $c_{k, j}=c_{k}$ for any $k>0,1 \leq j \leq n_{k}$, in Definition 3. For all $k>0, \sigma \in D_{k}$, $\operatorname{dist}\left(J_{\sigma * j}, J_{\sigma *(j+1)}\right)=\operatorname{dist}\left(J_{\sigma *(j+1)}, J_{\sigma *(j+2)}\right)\left(1 \leq j \leq n_{k+1}-2\right)$, and the left endpoint of $J_{\sigma * 1}$ is the left endpoint of $J_{\sigma}$ and the right endpoint of $J_{\sigma * n_{k+1}}$ is the right endpoint of $J_{\sigma}$. The set $E\left(\in M\left(J,\left\{n_{k}\right\},\left\{c_{k}\right\}\right)\right)$ is called the homogeneous Cantor set. Write $E=C\left(J,\left\{n_{k}\right\},\left\{c_{k}\right\}\right)$.
Theorem 4 (see [10]). Suppose $E=C\left(J,\left\{n_{k}\right\},\left\{c_{k}\right\}\right)$. Then,

$$
\begin{aligned}
\operatorname{dim}_{H} E & =\lim \inf _{k \rightarrow \infty} \frac{\log n_{1} n_{2}, \ldots, n_{k}}{-\log c_{1} c_{2}, \ldots, c_{k}}, \\
\operatorname{dim}_{P} E & =\overline{\operatorname{dim}}_{B} E=\lim \sup _{k \rightarrow \infty} \frac{\log n_{1} n_{2}, \ldots, n_{k+1}}{-\log c_{1} c_{2}, \ldots, c_{k}+\log n_{k+1}} .
\end{aligned}
$$

Theorem 5. Suppose $E=C\left(J,\left\{n_{k}\right\},\left\{c_{k}\right\}\right)$ If $\lim _{k \rightarrow \infty} n_{k}=\infty$, then $\operatorname{dim}_{A} E=1$.

Proof. Take $x$ as the left endpoint of some basic elements of order $k$ and $R=c_{1} c_{2}, \ldots, c_{k}$; then, $c_{1} c_{2}, \ldots, c_{k}$ is the length of basic elements of order $k$. It is obvious that $x \in E$. Notice that

$$
c_{1} c_{2}, \ldots, c_{k+1}+\frac{c_{1} c_{2}, \ldots, c_{k}\left(1-n_{k+1} c_{k+1}\right)}{n_{k+1}-1}=\frac{c_{1} c_{2}, \ldots, c_{k}\left(1-c_{k+1}\right)}{n_{k+1}-1} .
$$

Here, $c_{1} c_{2}, \ldots, c_{k+1}$ is the length of the basic elements of order $k+1$ of the Moran set, and $\left(\left(c_{1} c_{2}, \ldots, c_{k}\left(1-n_{k+1} c_{k+1}\right)\right) /\left(n_{k+1}-1\right)\right)$ is the length of the interval among the basic elements of order $k+1$ of the Moran set $E$. Take $r=\left(\left(c_{1} c_{2}, \ldots, c_{k}\left(1-c_{k+1}\right)\right) /\left(n_{k+1}-1\right)\right)$; then,

$$
N_{r}(B(x, R) \cap E) \geq n_{k}+1=\frac{\left(1-c_{k+1}\right) n_{k+1}}{n_{k+1}-1} \frac{R}{r} \geq \frac{1}{2} \frac{R}{r} .
$$

Note that $(R / r)=\left(\left(n_{k+1}-1\right) /\left(1-c_{k+1}\right)\right) \longrightarrow \infty$ when $k \longrightarrow \infty$; therefore, $\operatorname{dim}_{A} E=1$.

Theorem 6. Suppose $E=C\left(J,\left\{n_{k}\right\},\left\{c_{k}\right\}\right)$. If $\lim _{k \rightarrow \infty} n_{k} c_{k}=0$, then $\operatorname{dim}_{L} E=0$.

Proof. Take $x$ as the left endpoint of some basic elements of order $k+1$ and

$$
\begin{aligned}
R & =c_{1} c_{2}, \ldots, c_{k+1}+\frac{c_{1} c_{2}, \ldots, c_{k}\left(1-n_{k+1} c_{k+1}\right)}{n_{k+1}-1} \\
& =\frac{c_{1} c_{2}, \ldots, c_{k}\left(1-c_{k+1}\right)}{n_{k+1}-1} .
\end{aligned}
$$

Here, $c_{1} c_{2}, \ldots, c_{k+1}$ is the length of the basic elements of order $k+1$ of the Moran set and $\left(\left(c_{1} c_{2}, \ldots, c_{k}\left(1-n_{k+1} c_{k+1}\right)\right) /\left(n_{k+1}-1\right)\right)$ is the length of the interval among the basic elements of order $k+1$ of the Moran set $E$. Take $r=c_{1} c_{2}, \ldots, c_{k+1}$. It is obvious that

$$
N_{r}(B(x, R) \cap E) \leq 3=3\left(\frac{R}{r}\right)^{0} .
$$

Note that $(R / r)=\left(\left(1-c_{k+1}\right) /\left(\left(n_{k+1}-1\right) c_{k+1}\right)\right) \longrightarrow \infty$ when $k \longrightarrow \infty$; therefore, $\operatorname{dim}_{L} E=0$.

Example 1. Take $n_{k}=3^{k}$ and $c_{k}=4^{-k}$, and then $\operatorname{dim}_{L} E=0$, $\operatorname{dim}_{H} E=\operatorname{dim}_{P} E=\overline{\operatorname{dim}}_{B} E=(\log 3 / \log 4)$, and $\operatorname{dim}_{A} E=1$. 
Remark 3. By Theorem 5 and Theorem 6 , we give a negative answer to the conjecture in the paper [8, see Remark 1].

\section{Data Availability}

The data used to support the study are included within the article.

\section{Conflicts of Interest}

The authors declare that they have no conflicts of interest.

\section{Acknowledgments}

This work was supported by the Scientific Research Project of Hubei Provincial Department of Education (B2018358).

\section{References}

[1] P. Assouad, "Plongements lipschitziens dans $\mathbb{R}^{n}$," Bulletin De La Société Mathématique De France, vol. 111, pp. 429-448, 1983.

[2] D. G. Larman, "A new theory of dimension," Proceedings of the London Mathematical Society, vol. s3-17, no. 1, pp. 178192, 1967.

[3] L. Olsen, "On the Assouad dimension of graph directed Moran fractals," Fractals, vol. 19, no. 2, pp. 221-226, 2011.

[4] J. M. Mackay, "Assouad dimension of self-affine carpets," Conformal Geometry and Dynamics of the American Mathematical Society, vol. 15, no. 12, pp. 177-187, 2011.

[5] Z. Wen, "Moran sets and Moran classes," Chinese Science Bulletin, vol. 46, no. 22, pp. 1849-1856, 2001.

[6] W. Li, W. Li, J. Miao, and L. Xi, "Assouad dimensions of Moran sets and Cantor-like sets," Frontiers of Mathematics in China, vol. 11, no. 3, pp. 705-722, 2016.

[7] J. Li, "Assouad dimensions of Moran sets," Comptes Rendus Mathematique, vol. 351, no. 1-2, pp. 19-22, 2013.

[8] S. Hua, H. Rao, Z. Wen, and J. Wu, "On the structures and dimensions of Moran sets," Science in China Series A: Mathematics, vol. 43, no. 8, pp. 836-852, 2000.

[9] K. R. Pasatharathy, Probability Measures on Metric Spaces, Academic Press, New York, NY, USA, 1967.

[10] D. J. Feng, Z. Y. Wen, and J. Wu, "Dimensions of homogeneous Moran sets," Science in China Series A, vol. 27, pp. 1-7, 1997. 